\title{
Privatheit / Privacy
}

Liebe Leserinnen, liebe Leser,

Die beiden Begriffe werden analog verwendet. Alan Westin liefert zu "Privacy" eine häufig zitierte Definition: „Privacy is the right to be left alone". Entsprechend wäre im Deutschen Privatheit das Recht, in Ruhe gelassen zu werden. Das In-Ruhe-gelassen-werden reicht aber nicht aus, alles zu umfassen, was man unter Privatheit versteht; zum Beispiel schützt der Datenschutz die Privatheit von Daten aber nicht das Bedürfnis, in Ruhe gelassen zu werden. Auch für das englische "data privacy" trifft also die Definition nicht zu. Man muss das Schutzgut weiter fassen. Dann aber stellt sich leicht heraus, dass es letzten Endes die Privatheit ist, die geschützt werden soll. Privatheit das Recht auf Privatheit? Das geht nicht. Ein Recht kann nicht mit seinem Schutzgegenstand identisch sein. Privatheit ist kein Recht, sondern das Schutzgut eines Rechts. Sie ist etwas positiv Erfühltes und nicht das Recht auf seinen Schutz.

Das Bundesverfassungsgericht spricht in seinem Urteil zum Volkszählungsgesetz nicht von Privatheit, sondern von „Privatsphäre“. Es verzichtet auf eine Definition. Daneben akzeptiert es auch den von den Klägern genutzten, ebenfalls nicht definierten Begriff "Intimsphäre" als einen besonders schutzbedürftigen, von der Privatsphäre eingeschlossenen Bereich. Diese Vorstellung von Sphären, die konzentrisch angeordnet sind und eine von innen nach außen abnehmende Intensität von Privatheit aufweisen, ist zwar anschaulich; aber eine Sphäre ist kein geeigneter Schutzgegenstand für ein Recht. Sie ist ein abstrakter geometrischer Begriff und verleitet dazu, Privatheit als räumlich verteilt, etwa um die eigene Wohnung herum konzentriert zu verstehen. Das verfehlt aber das Gesuchte.

Der vom Recht gemeinte Bereich der Privatheit liegt nicht im dreidimensionalen natürlichen Raum. Er liegt vielmehr in derWelt gegenläufiger Interessen von Freiheit des Individuums und Macht der Gesellschaft. Seine Grenzen werden von der Gesellschaft gezogen. Sie kann grundsätzlich auch intime Daten ausschließen. Dafür gibt es Beispiele, etwa in der Medizin. Der räumliche Bezug von "Sphäre“ kann also irreführen. Die Westin'sche Definition vermeidet einen Raumbezug, indem sie privacy als ortsunabhängiges Recht definiert. Das mag ein Vorteil sein; es bleibt aber die oben gestellte Frage nach dem Schutzgegenstand dieses Rechts? Wenn das to-be-left-alone zu kurz greift, was ist es dann?

Was das ist, haben wir im Gefühl; es ist uns vertraut, wollen wir doch ein Recht darauf. Dazu folgendes: Bald nach dem Kriege, brachte das damalige Radio München eine viel gehörte Sendereihe. Sie war nach dem Schlager benannt, der jeweils zu Beginn einer Sendung auf sie einstimmte:„Ein warmer Ofen, ein Schalerl Kaffee ....". Sie stimmte das Lied vom häuslichen Glück an. Nach den Jahren in zugewiesenen Luftschutzkellern und des Dienstes am Gemeinwohl kam das Thema besonders gut an. Die Menschen waren von den eben vergangenen Jahren des Dienstes am Volk und der Geringachtung des Individuums erschöpft und freuten sich über eine gerettete Couch und ein Brikett im Bullerofen. Das ergab einen starken Eindruck. Privatheit bedeutete den Hörern der Sendung, unbelästigt und nach eigenem Gusto das Angenehme am häuslichen Leben genießen zu können. Eine Abschottung von der Öffentlichkeit, eine individuelle Freiheit, die sich mit einer abschließbaren Wohnung realisieren ließ.

Dass man sich ein Recht auf Privatheit ausrechnete, kam erst deutlich später, als sich die Menschen vom Staat und Wirtschaft mittels ihrer erfassten Daten bloßgestellt fühlten. Für sie war Privatheit etwas anderes als das häusliche Glück. Und diese Privatheit der Daten hinwiederum stellt sich heute, nach 40 Jahren Datenschutz, mit Internet und Big Data erheblich anders dar als die vom Anfang der 70er-Jahre.

Privatheit als Schutzgut eines Rechts, liebe Leserinnen und Leser, kann vielerlei sein. Sie ist immer etwas Erfühltes, in ihrem erfühlten Kern rational nicht Fassbares. Das und die Ansprüche darauf ändern sich mit den Zeiten. Und die Zeiten ändern sich mit uns.

Mit freundlichen Grüßen, Ihr

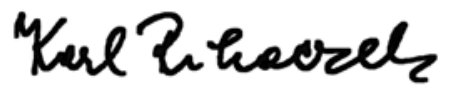

